\title{
Golgi apparatus-targeted AlEgens for effective photodynamic therapy through the crosstalk of Golgi apparatus and mitochondria
}

\section{Minglun Liu}

Nanjing University

Yuncong Chen ( $\nabla$ chenyc@nju.edu.cn )

Nanjing University https://orcid.org/0000-0002-8406-4866

\section{Yan Guo}

Nanjing University

Hao Yuan

Nanjing University

\section{Shankun Yao}

Nanjing University

\section{Suxing Jin}

Nanjing University

\section{Huanhuan Fan}

Hunan University

\section{Chengjun Wang}

Sinopec Shengli Petroleum Engineering Limited Company

\section{Weijiang He}

Nanjing University https://orcid.org/0000-0002-3157-5769

\section{Zijian Guo}

Nanjing University https://orcid.org/0000-0003-4986-9308

\section{Article}

Keywords: Golgi apparatus (GA), drug design, drug delivery, tumour growth

Posted Date: July 22nd, 2021

DOI: https://doi.org/10.21203/rs.3.rs-731967/v1

License: (c) (1) This work is licensed under a Creative Commons Attribution 4.0 International License. Read Full License 
Version of Record: A version of this preprint was published at Nature Communications on April 21st, 2022. See the published version at https://doi.org/10.1038/s41467-022-29872-7. 


\section{Abstract}

Golgi apparatus (GA) oxidative stress induced by in situ reactive oxygen species (ROS) could severely damage the morphology and function of GA, which may open up a new avenue for effective photodynamic therapy (PDT). However, due to the lack of effective design strategy, photosensitizers (PSs) with specific GA targeting ability have not been reported. Herein, we report aggregation-induced emission luminogen (AIEgen) based PSs that can effectively target to GA with Pearson correlation coefficient (PCC) up to 0.98 and singlet oxygen generation rate up to $77.8 \%$. GA fragmentation and cleavage of GA proteins (p115/GM130) were observed upon light irradiation. Meanwhile, the apoptotic pathway was activated through a crosstalk between GA oxidative stress and mitochondria in HeLa cells. Finally, TPE-PyT-CPS can effectively inhibited tumour growth in vivo with negligible adverse effect. This work provided a promising design strategy for the development of PSs with specific GA targeting ability, which is of great importance for precise and effective PDT.

\section{Introduction}

Photodynamic therapy (PDT) is an attractive tumor treatment which could be spatially and temporally controlled by light. ${ }^{[1]}$ It shows advantages such as minimal invasiveness, selective killing of tumor by light induced cytotoxic reactive oxygen species (ROS), particularly singlet oxygen $\left({ }^{1} \mathrm{O}_{2}\right)$, and repeated administration without drug resistance, which are problems related to the use of chemotherapeutic drugs (e.g., cisplatin). ${ }^{[2]}$ However, traditional photosensitizers (PSs) tend to show diminished ${ }^{1} \mathrm{O}_{2}$ quantum yield at aggregated states due to the enhanced non-radiative decay rate, which might decrease their PDT efficiency. ${ }^{[3-5]}$ In this regard, PSs with aggregation induced emission (AIE) characteristics are superior to traditional PSs since they show both increased ${ }^{1} \mathrm{O}_{2}$ and fluorescence quantum yield upon aggregation. ${ }^{[6-}$ ${ }^{7]}$ In addition, AIE luminogens (AIEgens) with intramolecular charge transfer (ICT) effect could facilitate the intersystem crossing (ISC) by decreasing $\Delta E_{\mathrm{ST}}$ (energy gap between the lowest singlet state $\mathrm{S}_{1}$ and the lowest triplet state $\mathrm{T}_{1}$ ), which could be adopted for developing heavy-atom-free PSs that show minimum dark toxicity. ${ }^{[8-9]}$ Thus, AlEgen based PSs could serve as appealing alternatives for traditional PSs and have attracted much attention for efficient PDT. ${ }^{[10-12]}$

Studies have revealed that ${ }^{1} \mathrm{O}_{2}$ shows very short lifetime (half-life: 0.03 to $0.18 \mathrm{~ms}$ ) and narrow diffusion distance in biological systems (a radius of $<0.02 \mu \mathrm{m}$ ). ${ }^{[1]}$ Hence, it is desirable to develop PSs that could target organelles and generate high dosage of ${ }^{1} \mathrm{O}_{2}$ in situ to direct cause dysfunction of subcellular organelles and activate specific cell death signals. Several PSs targeting mitochondria, ${ }^{[13-15]}$ endoplasmic reticulum (ER), ${ }^{[16-18]}$ |ysosome ${ }^{[19-20]}$ and cell membrane ${ }^{[21-22]}$ have been reported and exhibited high PDT efficiency. However, due to the lack of effective Golgi apparatus (GA) targeting strategies, specific GA targeting AIGgen based PSs have seldomly been reported. Golgi apparatus is a central node at the intersection point between the exocytic and endocytic channels in intracellular membrane transporting, which plays a pivotal role in the classification of newly synthesized and recycled 
proteins and lipids to their final destinations. ${ }^{[23]}$ Oxidative stress will induce significant damages on the structure and physiological function of GA. ${ }^{[24]}$ These changes in GA may trigger and propagate downstream stress signals, leading to GA disruption or even apoptosis. ${ }^{[25-28]}$ Therefore, GA targeting AlEgen based PSs could serve as good candidates for efficient PDT.

Herein, we report AIEgen based GA targeting PSs and their applications in efficient suppression of tumor cells by PDT induced GA oxidative stress (Figure 1A). Among the AIEgens, TPE-PyT-CPS showed a high ${ }^{1} \mathrm{O}_{2}$ quantum yield of $77.8 \%$ and excellent GA targeting ability with a Pearson's correlation coefficient (PCC) of 0.98. Structure-property relationship studies suggested that the cyano-group played a key role for specific GA targeting, while the strong ICT process and pyrene group contributed for high ISC rate and ${ }^{1} \mathrm{O}_{2}$ generation quantum yield. GA suffered from severe oxidative stress and fragmentation after ROS was generated in situ upon PDT. As shown in Figure 1B, the structural protein p115 of GA was found to be cleaved into $\mathrm{N}$-terminal and $\mathrm{C}$-terminal fragments, the latter then translocated into nucleus, resulting up regulation of p53 and dysfunction of mitochondria and activation of apoptotic pathway. Finally, prominent inhibition of tumor cell growth was demonstrated without noticeable adverse effect. This study not only provided an efficient strategy for developing GA targeted PSs, but also offered new insights for efficient and precise treatment of cancers.

\section{Results And Discussion}

\section{Design, synthesis, spectroscopic and ROS generation properties}

Traditional PSs usually have to face problems such as aggregation caused quenching (ACQ) and potential dark toxicity due to heavy atom modification. ${ }^{[29-30]}$ AIEgen based PSs with a heavy atom free D$\pi-A$ structure can efficiently separate the highest occupied molecular orbital (HOMO) and lowest unoccupied molecular orbital (LUMO) distribution, which is helpful to promote the rate of intersystem crossing $\left(k_{\text {ISC }}\right)$ process and thus beneficial to increase ${ }^{1} \mathrm{O}_{2}$ generation. ${ }^{[30]}$ TPE-PyT-CPS was designed with an AIE active tetraphenylethene (TPE) derivative as the electron donating group (EDG) and a cyanopyridinium salt moiety act as the electron withdrawing group (EWG). To improve the ISC efficiency, a pyrene ring and thiophene group was introduced as $\pi$ spacer for better separation of HOMO-LUMO and decreasing $\Delta E_{\mathrm{ST}}$. The synthesis procedures of TPE-PyT-CPS and its control compounds were presented in Supporting Information. All the intermediates and target compounds were fully characterized by ${ }^{1} \mathrm{H}$ and ${ }^{13} \mathrm{C}$ NMR spectroscopy and HRMS.

The spectroscopic properties of all compounds in water were studied with the UV-vis and photoluminescence (PL) spectrometer (Figure S17), in which the TPE-PyT-CPS shows a maximum absorption peak at $498 \mathrm{~nm}$ and an NIR emission at $693 \mathrm{~nm}$, respectively. The Stokes shift was nearly 200 $\mathrm{nm}$, which can reduce the interference of background signal and helps to improve the image-guided therapy. Moreover, compared with the TPE-PyT-PS and TPE-PyT-CP, the UV absorption spectra and fluorescence emission spectra of TPE-PyT-CPS and TPE-T-CPS show a significant red shift, which is due 
to the stronger ICT effect caused by EDGs of cyano-group and pyridinium salt group. As depicted in Figure 2A and B, TPE-PyT-CPS in pure acetonitrile exhibited very weak emission, while with the water fraction increasing to $30 \%$, the emission enhanced slightly. Further increasing the water fraction to $99 \%$, lead to a distinct enhancement on PL intensity with a gradual red shift of the emission maximum to 680 $\mathrm{nm}$, indicating typical AIE characteristics of TPE-PyT-CPS. The fluorescence quantum yields of TPE-PyTCPS in acetonitrile and water (with $1 \%$ acetonitrile, $v / v$ ) were $2.74 \%$ and $17.1 \%$, respectively. In addition, The AIE features of the other three AIEgens were also confirmed using a mixed solvent (acetonitrile and water) system with different water fractions (Figure S18, Table S1).

Next, dynamic light scattering (DLS) tests showed that the particle sizes of TPE-PyT-CPS in water and acetonitrile were around $410 \mathrm{~nm}$ and $1 \mathrm{~nm}$, respectively (Figure $2 \mathrm{C}$ ). The results suggested that the TPEPyT-CPS is almost monodisperse in acetonitrile but form aggregates (rod-like according to TEM) in aqueous solution. The in vitro ${ }^{1} \mathrm{O}_{2}$ generation capability of TPE-PyT-CPS was evaluated under $532 \mathrm{~nm}$ laser irradiation with 9,10-anthracenediyl-bis(methylene)dimalonic acid (ABDA) as the ${ }^{1} \mathrm{O}_{2}$ indicator (Figure 2D-F). Upon light irradiation, the absorbance of ABDA in acetonitrile showed almost no change within 12 minutes in the presence of TPE-PyT-CPS, while in water (contains $1 \%$ acetonitrile) it decreased sharply within 150 seconds, confirming that TPE-PyT-CPS possessed a dramatically elevated ROS in aggregation state.

The ${ }^{1} \mathrm{O}_{2}$ quantum yield $\left(\Phi_{\Delta}\right)$ of TPE-PyT-CPS in aqueous solution was determined to be $77.8 \%$, while those of the control compounds were found to be $71.4 \%, 62.7 \%$ and $19.2 \%$ for TPE-T-CPS, TPE-PyT-PS and TPE-PyT-CP, respectively (Figure S19, Table S1). TPE-PyT-PS showed much higher $\Phi_{\Delta}$ than TPE-PyT$\mathrm{CP}$, suggesting that the pyridinium group contributed more for ROS generation than the $-\mathrm{CN}$ group. The stronger electron withdrawing ability of pyridinium group than $-\mathrm{CN}$ group lead to stronger ICT effect and more efficient ISC of TPE-PyT-PS than TPE-PyT-CP. It is also reasonable that TPE-PyT-CP and TPE-PyT-PS showed lower ROS generation yields than TPE-PyT-CPS and TPE-T-CPS, since the former two showed weaker ICT effects due to the lack of EDGs of either pyridinium group or - $\mathrm{CN}$ group.

To understand the mechanism of higher ROS generation ability of TPE-PyT-CPS than that of TPE-T-CPS, which showed identical electron donor and acceptor but different $\pi$ spacers, density functional theory (DFT) calculation was conducted. As shown in Figure 3A, although the HOMO and LUMO electronic distributions of TPE-T-CPS are well separated, there are some overlaps at the region of thiophene and the nearby phenyl ring of TPE-T-CPS. However, by introducing an additional large $\pi$ spacer pyrene, the overlap between HOMO and LUMO of TPE-PyT-CPS was distinctly reduced. As a result, the $\triangle E_{\mathrm{ST}}$ value of TPEPyT-CPS was calculated to be $0.20 \mathrm{eV}$, smaller than that of TPE-T-CPS, which was calculated $0.39 \mathrm{eV}$ (Figure 3B, Table S2). The DFT calculation was consistent with the experimental data that TPE-PyT-CPS showed higher ${ }^{1} \mathrm{O}_{2}$ quantum yield than TPE-T-CPS in water, which verified our design strategy that the introduction of pyrene spacer can help improve the HOMO-LUMO separation within the ICT system, thus elevate the ${ }^{1} \mathrm{O}_{2}$ generation ability. 


\section{Subcellular localization study}

The intracellular localization of TPE-PyT-CPS was investigated by confocal laser scanning microscopy (CLSM). As shown in Figure 4, the Pearson's correlation coefficients (PCCs) between fluorescence images of marked commercial dyes and the TPE-PyT-CPS were determined to be 0.36 for Mito, 0.54 for ER, 0.98 for GA and 0.61 for lysosome, respectively. The results indicated that TPE-PyT-CPS localized preferentially in GA with high specificity. It was strange that TPE-Py-CPS showed minimum localization in mitochondria, although it contained a cationic pyridinium group. Therefore, the structure-property relationships of the four compounds were investigated to figure out which moiety was responsible for the GA targeting ability (Figure 1A). The PCC values between the fluorescence images of GA marker and those of each compound were determined to be $0.98,0.90,0.89$ and 0.68 for TPE-PyT-CPS, TPE-T-CPS, TPE-PyT-CP and TPE-PyT-PS, respectively (Figure 4, Figure S21-S23). We also tested the lipophilicity of all compounds (Figure S20, Table S2) because lipophilicity was reported to play an important role in the cell uptake and distribution of compounds in subcellular organelles. ${ }^{[31]}$ The $\log P_{\mathrm{O} / \mathrm{W}}$ values of each compound were determined to be 1.764, 1.582, 2.707 and 2.215 for TPE-PyT-CPS, TPE-T-CPS, TPE-PyT-CP and TPE-PyT-PS, respectively. There seemed to be no direct link between lipophilicity and GA targeting ability of these AlEgens. However, it was obvious that the absence of the -CN group in TPE-PyT-PS made it a poor GA targeting dye, indicating that the $-\mathrm{CN}$ group played an essential role for GA targeting in this AlEgen system.

\section{ROS generation and PDT in tumor cells}

The high ${ }^{1} \mathrm{O}_{2}$ quantum yield and excellent GA targeting ability of TPE-PyT-CPS encouraged us to investigate its ability to kill cancer cells through PDT. The in situ ${ }^{1} \mathrm{O}_{2}$ generation capability was evaluated by detecting the fluorescence intensity of Singlet Oxygen Sensor Green (SOSG). As shown in Figure 5A, TPE-PyT-CPS and TPE-T-CPS treated HeLa cells showed strong green fluorescence under laser irradiation, while almost no fluorescence was observed under dark condition. Under the same condition, SOSG signal of cells stained with TPE-PyT-CPS was higher than that of TPE-T-CPS treated cells. This was in good accordance with the fact that TPE-PyT-CPS has higher singlet oxygen generation capacity. Subsequently, the PDT effects of TPE-PyT-CPS and TPE-T-CPS were evaluated by 3-(4,5-dimethy Ithiazol-2-yl)-2,5diphenyltetra-zolium bromide (MTT) assay. Upon $532 \mathrm{~nm}$ laser irradiation $\left(65 \mathrm{~mW} \mathrm{~cm}^{-2}\right)$ for $2 \mathrm{~min}$, dosedependent toxicities were observed and half maximal inhibitory concentrations $\left(\mathrm{IC}_{50}\right)$ to HeLa cells were determined to be $170 \mathrm{nM}$ and $400 \mathrm{nM}$ for TPE-PyT-CPS and TPE-T-CPS, respectively (Figure 5B). However, negligible dark toxicities were observed upon incubation of HeLa cells with TPE-PyT-CPS and TPE-T-CPS (up to $256 \mu \mathrm{M}$ ) for $24 \mathrm{~h}$, demonstrating exceptionally high phototoxicity index ( $\mathrm{PI}=\mathrm{IC} \mathrm{I}_{50}$ dark/ $/ \mathrm{IC}_{50}$ light) values over 1500 and 640 (Figure 5 C). The results suggested that TPE-PyT-CPS and TPE-T-CPS could effectively generate ROS and suppress cancer cell growth. Since TPE-PyT-CPS showed better PDT performance in vitro, it was selected for the following mechanistic and in vivo PDT studies. 
To study the cell death mechanism of tumor cells, Annexin V-FITC and propidium iodide (PI) were used to monitor the process of cell apoptosis induced by TPE-PyT-CPS mediated PDT. HeLa cells were incubated with TPE-PyT-CPS and irradiating with $532 \mathrm{~nm}$ laser $\left(65 \mathrm{~mW} \mathrm{~cm}^{-2}, 2 \mathrm{~min}\right)$, followed by Annexin V-FITC and PI staining, then the fluorescence images were collected using CLSM. As shown in Figure 5D, the green and red fluorescence signals were hardly detected with irradiation alone or with TPE-PyT-CPS but without irradiation. However, after TPE-PyT-CPS incubation and light irradiation for 2 minutes, distinct green and red fluorescence were observed in HeLa cells, suggesting the occurrence of cell apoptosis. In addition, compared with the control group, the cells treated with TPE-PyT-CPS and light irradiation, showed significant shrinkage as indicated by the white arrow in the bright fields. Moreover, flow cytometry analysis demonstrated that when the apoptosis rate of HeLa cells reached $56.7 \%$ when incubated with TPE-PyT-CPS $(0.2 \mu \mathrm{M})$ under light irradiation (Figure S24). The results indicated that apoptosis was the main death pathway of tumor cells upon PDT treatment.

\section{PDT induced GA morphology change}

TPE-PyT-CPS was confirmed to target GA specifically and generate abundant ROS in situ, which could induce severe damage on structural proteins of GA and cause morphological and functional changes of GA. ${ }^{[32-33]}$ Therefore, we used CLSM and TEM to monitor the ultrastructural alterations of GA in HeLa cells caused by TPE-PyT-CPS (Figure 6). When the cells were treated with light only or with TPE-PyT-CPS (0.2 $\mu \mathrm{M})$ in the dark, negligible effect on the GA morphology was observed. However, when TPE-PyT-CPS $(0.2$ $\mu \mathrm{M}$ ) and light were present (i.e., PDT) in unison, the Golgi structure of HeLa cells changed obviously, including intensive swelling and fragmentation of GA cistern. The results confirmed that TPE-PyT-CPS could cause severe damage on GA structure by in situ ${ }^{1} \mathrm{O}_{2}$ generation during PDT.

\section{GA oxidative stress caused apoptosis pathway}

The morphological changes of Golgi apparatus can activate the related signal pathways and trigger cell repair or apoptosis. ${ }^{[34]}$ P115 is a vesicle tethering protein, which functions in Golgi-vesicle tethering and Golgi-cisternal stacking by bridging GM130 and giantin. ${ }^{[35]}$ To further ascertain whether these structural proteins were affected during GA-targeted PDT process, the expressions of p115 and GM130 in HeLa cells were evaluated by western blot. As shown in Figure 7A and B, the expression of GM130 and p115 in HeLa cells was gradually decreased with the extension of irradiation time during PDT, especially for GM130. In addition, we found two new fragments of $30 \mathrm{kD}$ (C-terminal fragment) and $90 \mathrm{kD}$ (N-terminal fragment) proteins which originated from p115 during this process, confirming that p115 was cleaved after GA oxidative stress. Moreover, the western blot experiments demonstrated that p53 and its phosphorylation derivative (Ser15) were significantly upregulated, which suggested that PDT induced GA stress was related to apoptosis.

Studies have suggested that the $30 \mathrm{kD}$ residue of $\mathrm{p} 115$ can translocate into the nucleus through sumoylation and then interact with p53, leading to p53 phosphorylation, which leads to apoptosis pathway. ${ }^{[36-37]}$ To verify this mechanism, we detected the level of p53 upregulated modulator of 
apoptosis (PUMA), which was located in the outer membrane of the mitochondria. ${ }^{[38]}$ Indeed, the dramatically increased expression of pro-apoptotic protein PUMA and Bax was observed, accompanied with a down regulation of anti-apoptotic Bcl-2 in HeLa cells during PDT, resulting an increased Bax/Bcl-2 ratio (Figure 7C, D). In addition, an obvious upregulation of cleaved caspase-3 (a mitochondria-apoptotic protein) was also observed.

The activation of Bax can cause its conformational change and then oligomerization in the outer membrane of mitochondria, which will reduce the mitochondrial membrane potential $\left(\mathrm{MMP}, \Delta \psi_{\mathrm{m}}\right) \cdot{ }^{[39-40]}$ Next, the MMP was evaluated through JC-1 staining assay. As shown in Figure 8, the HeLa cells after PDT treatment showed an evident increase in green fluorescence of JC-1 monomers and a significant decrease in red channel of JC-1 aggregates, suggesting a distinct loss of MMP upon PDT. However, HeLa cells treated by light only, dark and "dark + AIEgen" showed intense red fluorescence and weak green fluorescence, indicating intact MMPs. The above results suggested that PDT induced GA oxidative stress could severely affect mitochondria homeostasis and active the apoptosis signal pathway (Figure 9).

\section{GA-targeting mediated PDT in vivo}

The in vivo PDT performance of TPE-PyT-CPS was evaluated in a subcutaneous HeLa tumor-bearing mouse model. All animal experiments were carried out in accordance with the regulations of the Institutional Animal Care and Use Committee (IACUC) of Nanjing University. Firstly, we evaluated the imaging ability of TPE-PyT-CPS in mice (Figure 10A) and monitored the fluorescence signals at specified intervals. Distinct fluorescence signal in the tumor sites was observed after intratumoral injection of TPEPyT-CPS (0.1 mM, $120 \mu \mathrm{L} / 200 \mathrm{~mm}^{3}$ tumor) for 3 hours (Figure S25), and the fluorescence intensity reached maximum after 18 hours. To gain the fluorescence distribution image of the tumor and other major organs, the mice were sacrificed at $30 \mathrm{~h}$ after injection of TPE-PyT-CPS, and the ex vivo fluorescence images of isolated organs were shown in Figure S25. The results showed that TPE-PyT-CPS could effectively retain in the tumor tissue and exhibit strong fluorescence signal. Moreover, there was almost no fluorescence signals originated from other major organs, including heart, spleen, lung and kidney, except for a weak fluorescence in liver tissue. The results demonstrated that TPE-PyT-CPS could retain in tumor area and serve as an imaging agent.

Subsequently, the anticancer efficacy of TPE-PyT-CPS was assessed in vivo. Tumor (HeLa) bearing Balb/c mice were randomly divided into four groups $(n=5)$ : saline group (saline light - ), saline group with laser irradiation group (saline light +), AlEgen group (AIEgen light -) and AIEgen with laser irradiation group (AlEgen light + ). After intratumoral injection of TPE-PyT-CPS $(0.2 \mathrm{mM}, 100 \mu \mathrm{L})$ for $18 \mathrm{~h}$, the tumor area of the mice was irradiated with $532 \mathrm{~nm}$ laser of $35 \mathrm{~mW} \mathrm{~cm}^{-2}$ for $5 \mathrm{~min}$, labeled as AlEgen (light +) groups. As can be seen from Figure 10B, for the control groups (saline light -, saline light+), the tumor volumes increased rapidly regardless of light or dark conditions, suggesting that the tumor growth was not affected in the absence of TPE-PyT-CPS. However, in the presence of our AIEgen, the tumor volumes and weight were effectively suppressed in the light group (Figure 10C, D). Additionally, the body weights of mice in "AlEgen light +" group showed no obvious difference from those of control group during PDT 
(Figure 10E), which clearly demonstrated the minimal side effects of "AlEgen light + " treatment. To understand and verify the different antitumor efficacy and whether TPE-PyT-CPS cause in vivo side toxicity of those treatments, tumors and major organs from the four groups are excised and appraised using H\&E (hematoxylin and eosin) staining and TUNEL (terminal deoxynucleotidyl transferase mediated dUTP-biotin nick end labeling) assay for histological analysis. ${ }^{[41]}$ The staining results showed that the TPE-PyT-CPS treated group with laser irradiation (AIEgen light + ) exhibited obvious cell apoptosis with pyknotic cells with condensed nuclei (Figure 10F, Figure S26), whereas the tumors tissues of mice that treated in control groups (AIEgen light -, saline + light and saline) had massive viable cancer cells. Similar results were obtained in the TUNEL assay, the tumors slice of mice treating with PDT exhibited significantly higher level of cells apoptosis than the control group. Meanwhile, there was no abnormal pathological morphology or prominent tissue damage in the heart, liver, spleen, lung and kidney of mice in all treatment groups (Figure S27). Taken together, these results confirmed that TPE-PyT-CPS could efficiently suppress tumor growth by GA targeting mediated-PDT with negligible side effects.

\section{Conclusions}

In conclusion, we have designed and synthesized AlEgen based PSs with excellent GA targeting ability and PDT effect. Structure-property relationship studies revealed that the cyano-group played a decisive role in GA targeting, while the introduction of large $\pi$ spacer pyrene group further enhanced the singlet oxygen quantum yield of the $D-\pi-A$ system. Large morphological change of GA was observed upon the in situ generation of ROS during PDT. Moreover, we found the GA stress can trigger the mitochondria dysfunction during PDT. The GA-mitochondria crosstalk lead to the collapse of MMP and ultimately cell death. TPE-PyT-CPS showed excellent phototoxicity and negligible dark toxicity to HeLa cancer cells with a large PI over 1500. Finally, benefit from its high singlet oxygen generation and effective GA targeting features, TPE-PyT-CPS significantly inhibited the tumor growth of mice without obvious adverse effects on normal tissues in vivo after PDT. This work provided a reliable design strategy for the development of AlEgen based GA targeting PSs, which offered a new avenue for precise and efficient PDT through transferring stress signals from Golgi apparatus to mitochondria.

\section{Methods}

Materials and instrument. All chemicals for compounds synthesis were commercially available (SigmaAldrich, J\&K, Sinopharm, Bidepharma-Tech) and used without further purification. The silica gel (300-400 mesh) was used for column chromatography. The ${ }^{1} \mathrm{H}$ and ${ }^{13} \mathrm{C}$ NMR spectra were recorded on a Bruker DRX $400 \mathrm{MHz}$ or $600 \mathrm{MHz}$ spectrometer. ESI-MS was determined on a LCQ electrospray mass spectrometer (Thermo Finnigan). High resolution mass spectra (HR-MS) were determined using an Agilent 6540Q-TOF HPLC-MS spectrometer. UV-visible absorption spectra were recorded on a PerkinElmer Lambda 35 spectrophotometer. The transmission electron microscopy (TEM) images were obtained using a JEOL JEM-1011 transmission electron microscope (Japan). Fluorescence confocal imaging was carried out on a laser scanning confocal imaging system (Olympus TH4-200) consisting of ZEISS Laser 
Scanning Microscope (LSM 710) and a 20-mW output $488 \mathrm{~nm}$ argon ion laser. Flow cytometry analysis was performed with a BD LSRFortessa Cell Analyzer.

Density functional theory (DFT) calculation. Theoretical calculations were adopted to rationalize the ISC process of the TPE-PyT-CPS and TPE-T-CPS using Gaussion 09w. The geometries of TPE-PyT-CPS and TPE-T-CPS were optimized based on the method TD-DFT//B3LYP/6-31G(d). TD-DFT was used to predict the excitation energies for the singlet and triplet excited states of TPE-PyT-CPS and TPE-T-CPS in tetrahydrofuran.

Morphology and Size. Dynamic light scattering (DLS) was used to monitor the size of the TPE-PyT-CPS in water, acetonitrile solution and the morphology was observed by Transmission Electron Microscope (TEM).

Singlet oxygen detection in solution. ABDA (9,10-Anthracenediyl-bis(methylene)-dimalonic acid) was used as an indicator to detect singlet oxygen production capacity since the absorbance of ABDA decreases upon reaction with singlet oxygen, and RB (Rose Bengal) was used as an internal reference. For singlet oxygen detection, the ABDA $(50 \mu \mathrm{M})$ was mixed with the TPE-PyT-CPS in acetonitrile/water $(1: 99, v / v)$ and exposed to $532 \mathrm{~nm}$ laser irradiation $\left(10 \mathrm{~mW} \mathrm{~cm}^{-2}\right)$. The decomposition of ABDA was monitored by the absorbance decrease at $378 \mathrm{~nm}$. The ${ }^{1} \mathrm{O}_{2}$ quantum yield of the PS in water was calculated using the following formula (Eq. 1):

\section{$\Phi_{\mathrm{PS}}=\Phi_{\mathrm{RB}} \frac{K_{P S} A_{R B}}{K_{\mathrm{B} D A \mathrm{~A}}}$}

where $\mathrm{K}_{\mathrm{PS}}$ and $\mathrm{K}_{\mathrm{RB}}$ are the decomposition rate constants of $\mathrm{ABDA}$ by the TPE-PyT-CPS and RB, respectively. $A_{P S}$ and $A_{R B}$ represent the light absorbed by the different AIEgens (TPE-PyT-CPS, TPE-PyT-CP, TPE-PyT-PS and TPE-T-CPS) and RB, respectively, which are determined by integration of the areas under the absorption bands in the wavelength range of $400-600 \mathrm{~nm}$. $\Phi_{\mathrm{RB}}$ is the ${ }^{1} \mathrm{O}_{2}$ quantum yield of $\mathrm{RB}$, which is 0.75 in water.

Lipophilicity of AlEgens. Solutions of different AlEgens (concentration:15, 30, $45 \mu \mathrm{M}$ ) were prepared in phosphate buffer $(10 \mathrm{mM}, \mathrm{pH}$ 7.4) pre-saturated with 1-octanol. Equal volumes (1.5 mL) of the solution and 1-octanol pre-saturated with the phosphate buffer were mixed and placed in a thermostatic (25.0 \pm $0.1^{\circ} \mathrm{C}$ ) air bath orbital shaker at $200 \mathrm{rpm}$ for $4 \mathrm{~h}$. The samples were separated into two phases after centrifugation at $2500 \mathrm{rpm}$ for $15 \mathrm{~min}$. The concentration of the solute in the aqueous phase was determined by spectrophotometry. According to the law of mass conservation, the drug concentration of the 1-octanol phase and the lipohydro partition coefficient $P_{\mathrm{O} / \mathrm{W}}\left(P_{\mathrm{O} / \mathrm{W}}=C_{0} / C_{\mathrm{W}}=A_{0} / A_{\mathrm{W}}\right.$, A stands for absorbance) were calculated. The average of three parallel experimental data was reported as the final result. The $\log P_{\mathrm{O} / \mathrm{w}}$ was calculated using the following equation (Eq. 2):

$$
\log P_{\text {OrW }}=\log \left[\left([\mathrm{C}]_{\text {initial }}-[\mathrm{C}]_{\text {final }}\right)[\mathrm{C}]_{\text {final }}\right] \quad \text { Eq. } 2
$$


Subcellular organelle imaging. The HeLa cancer cells were used in the following experiments and cultured using DMEM culture medium containing 10\% FBS and $1 \%$ penicillin-streptomycin in an artificial environment $\left(5 \% \mathrm{CO}_{2}\right.$ at $\left.37^{\circ} \mathrm{C}\right)$. The HeLa cells were regularly checked for mycoplasma contamination and then conducted when the cells were grown to $80 \%$ confluence in the culture dish. The HeLa cancer cells were cultured in the special confocal chambers at a density of $10^{5}$ cells $\mathrm{mL}^{-1}$ in culture medium. After $24 \mathrm{~h}$, when cells have reached the desired confluence TPE-PyT-CPS $(10 \mu \mathrm{M})$, TPE-PyT-CP $(10 \mu \mathrm{M})$, TPE-T-CPS $(10 \mu \mathrm{M})$ and TPE-PyT-PS $(10 \mu \mathrm{M})$ in FBS free medium were added and incubated with HeLa cancer cells at $37^{\circ} \mathrm{C}$ for $6 \mathrm{~h}$.

Mitochondrial Imaging. The cells were then washed three times carefully using $1 \times$ PBS, followed by addition of commercial Mito Tracker Green FM (50 nM) and incubation for another 30 min. Subsequently, the cells were washed three times using $1 \times P B S$ and visualized by confocal laser scanning microscopy at $488 \mathrm{~nm}$ excitation for TPE-PyT-CPS, TPE-PyT-CP, TPE-T-CPS, TPE-PyT-PS $(10 \mu \mathrm{M})$ and $488 \mathrm{~nm}$ excitation for Mito Tracker Green FM, respectively. The signal collections of CLSM were $600-750 \mathrm{~nm}$ for different AlEgens and 495-535 nm for Mito Tracker Green.

Lysosome imaging. The cells were then washed three times carefully using $1 \times$ PBS, followed by addition of commercial LysoSensor ${ }^{\mathrm{TM}}$ Green DND-189 $(75 \mathrm{nM})$ and incubation for another 60 min under growth conditions appropriate for the particular cell type. Subsequently, the cells were washed three times using $1 \times$ PBS and visualized by confocal laser scanning microscopy at $488 \mathrm{~nm}$ excitation for TPE-PyT-CPS, TPE-PyT-CP, TPE-T-CPS, TPE-PyT-PS $(10 \mu \mathrm{M})$ and $488 \mathrm{~nm}$ excitation for LysoSensor ${ }^{\mathrm{TM}}$ Green DND-189, respectively. The signal collections of CLSM were $600-750 \mathrm{~nm}$ for different AlEgens and 495-535 nm for LysoSensor ${ }^{\mathrm{TM}}$ Green DND-189.

Golgi apparatus imaging. The cells were then washed three times carefully using $1 \times \mathrm{PBS}$, followed by addition of commercial Golgi Green $(5 \mu \mathrm{M})$ and incubation for 30 min under $4{ }^{\circ} \mathrm{C}$. After, washing the cells three times using $1 \times$ PBS growth and incubation for another 30 min under $37^{\circ} \mathrm{C}$. Subsequently, visualized by confocal laser scanning microscopy at $488 \mathrm{~nm}$ excitation for TPE-PyT-CPS, TPE-PyT-CP, TPE-T-CPS, TPE-PyT-PS $(10 \mu \mathrm{M})$ and $488 \mathrm{~nm}$ excitation for Golgi Green, respectively. The signal collections of CLSM were $600-750 \mathrm{~nm}$ for different AlEgens and 495-535 nm for Golgi Green.

Endoplasmic reticulum imaging. The cells were then washed three times carefully using $1 \times$ PBS, followed by addition of commercial ER-Tracker ${ }^{\mathrm{TM}}$ Blue $(1 \mu \mathrm{M})$ and incubation for another 45 min under growth conditions $\left(37^{\circ} \mathrm{C}\right)$ appropriate for the particular cell type. Subsequently, the cells were washed three times using $1 \times$ PBS and visualized by confocal laser scanning microscopy at $488 \mathrm{~nm}$ excitation for TPE-PyTCPS, TPE-PyT-CP, TPE-T-CPS and TPE-PyT-PS $(10 \mu \mathrm{M})$ and $405 \mathrm{~nm}$ excitation for ER-Tracker ${ }^{\text {TM }}$ Blue, respectively. The signal collections of CLSM were $600-750 \mathrm{~nm}$ for different AlEgens and 455-520 nm for ER-Tracker ${ }^{\mathrm{TM}}$ Blue. 
Intracellular ROS detection. The capability of TPE-PyT-CPS and TPE-T-CPS for intracellular ROS production were assessed using singlet oxygen sensor green (SOSG) as an ROS indicator. The HeLa cancer cells were cultured in the special confocal chambers and incubated with TPE-PyT-CPS $(0.2 \mu \mathrm{M})$ or TPE-T-CPS $(0.4 \mu \mathrm{M})$ for $6 \mathrm{~h}$ at $37^{\circ} \mathrm{C}$. Subsequently, the cells were washed three times with $1 \times$ PBS, followed by addition of singlet oxygen sensor green (SOSG, $15 \mu \mathrm{M}$ ) in FBS free culture medium and incubation for $30 \mathrm{~min}$. The above-mentioned process was performed in dark. Then, the cells were exposed to white light irradiation $\left(25 \mathrm{~mW} \mathrm{~cm}^{-2}\right)$ for $2 \mathrm{~min}$, followed by imaging with CLSM. For CLSM imaging, the excitation of SOSG was $488 \mathrm{~nm}$ with a collection of fluorescence signal at $525 \pm 20 \mathrm{~nm}$.

Cytotoxicity Study. Briefly, $100 \mu \mathrm{L}$ of HeLa cell suspension were added into each well of 96-well plate at a density of $8 \times 10^{4}$ cells $\mathrm{mL}^{-1}$. After $80 \%$ confluence, fresh culture medium (the concentration of DMSO lower than $0.5 \%$ ) containing a series of concentrations of TPE-PyT-CPS was added and incubated with the HeLa cancer cells for $6 \mathrm{~h}$ at $37^{\circ} \mathrm{C}$ in dark. Subsequently, the cells were exposed to $532 \mathrm{~nm}$ laser irradiation $\left(65 \mathrm{~mW} \mathrm{~cm}^{-2}\right)$ for $2 \mathrm{~min}$. Alternatively, the TPE-PyT-CPS-treated HeLa cancer cells were kept in dark without light exposure. At $24 \mathrm{~h}$ post irradiation, the wells were replaced with freshly prepared 3-(4,5dimethylthiazol-2-yl)-2,5-diphenyl tetrazolium bromide (MTT; $2.5 \mathrm{mg} \mathrm{mL}^{-1}$ in PBS) solution. After $4 \mathrm{~h}$ incubation, the solution in each well was carefully removed and $150 \mu \mathrm{L}$ of DMSO was added to each well to dissolve the formazan. The plate was gently shaken for $10 \mathrm{~min}$ at room temperature and then the absorbance of MTT at $595 \mathrm{~nm}$ was monitored by the microplate reader (Thermo Scientific Varioskan Flash) in order to determine the cell viability. Cell viability rates (\%) and $\mathrm{IC}_{50}$ values were calculated on the data of three parallel tests.

Annexin V-FITC/Propidium lodide Co-Staining Assay. HeLa cells pretreated with TPE-PyT-CPS $(0.2 \mu \mathrm{M})$ for 30 min were stained with both annexin V-FITC and PI following the standard protocol (Life Technologies) and imaged after irradiation of $532 \mathrm{~nm}$ laser $\left(65 \mathrm{~mW} \mathrm{~cm}^{-2}, 2 \mathrm{~min}\right)$. For control experiments, HeLa cells preincubated with TPE-PyT-CPS $(0.2 \mu \mathrm{M})$, annexin V-FITC, and PI were imaged without light irradiation. The excitation wavelengths for annexin V-FITC and PI were 488 and $559 \mathrm{~nm}$, respectively, and the emissions for annexin VFITC and PI were collected in the range of 500-530 and $600-630 \mathrm{~nm}$, respectively.

Detection the expression of p115, GM130, P53, ser15, PUMA, Bcl-2, Bax and Caspase-3. The HeLa cancer cells were seeded into $6 \mathrm{~cm}$ plates at the density of $1 \times 10^{5}$ cells $\mathrm{mL}^{-1}$. After adherence, the HeLa cancers cells were incubated with $0.2 \mu \mathrm{M}$ of TPE-PyT-CPS, for $6 \mathrm{~h}$ at $37^{\circ} \mathrm{C}$, respectively. For the dark control group, there is no need to light, and for the light control group, there is no need to add photosensitizer, only light for 20 minutes $\left(25 \mathrm{~mW} \mathrm{~cm}^{-2}\right)$. Next, the treated cells were washed and irradiated by $532 \mathrm{~nm}$ laser irradiation for 4, 6, 8, 10 and 20 min. After $6 \mathrm{~h}$, the cells were collected and then conducted with centrifugation using $12,000 \mathrm{rpm}$ for $15 \mathrm{~min}$ at $4{ }^{\circ} \mathrm{C}$. Protein concentrations were measured using protein assay reagents, and equal amounts of protein per lane were separated on SDS-PAGE gel and transferred to a PVDF membrane. The membrane was incubated with Anti-p115-RhoGEF (1:100, Abcam, ab223759), Anti-GM130 (1:1500, Abcam, ab52649), Anti-p53 (1:2000, Abcam, ab26), Anti-p53 (phospho S15) (1:2000, 
Abcam, ab26), Anti-PUMA (1:2000, Abcam, ab33906), Bcl-2 (1:200, Abcam, ab182858), Bax (1:5000, Abcam, ab32503), caspase-3 (1:200, Abcam, ab184787) and Anti-GAPDH (1:10000, Abcam, ab8245) followed by incubation with the peroxidase-labeled goat antirabbit HRP secondary antibody. Western blots were visualized by enhanced chemiluminescence detection system. Relative grayscale was calculated by ImageJ.

Mitochondrial membrane potential (MMP) assay. MMP was evaluated by confocal imaging via JC-1 staining. HeLa cells were seeded in a glass bottom cell culture dish at $40 \%$ confluence and cultured in media containing TPE-PyT-CPS $(0.2 \mu \mathrm{M})$ respectively. After the incubation of $6 \mathrm{~h}$, then cells were stained with JC-1 (Beyotime Biotechnology) following the manufacture's protocol. After rinse three times with incomplete culture medium, the cells irradiated with a laser of $532 \mathrm{~nm}\left(25 \mathrm{~mW} \mathrm{~cm}{ }^{-2}, 2 \mathrm{~min}\right)$, and confocal imaging was immediately carried out with Leica SP8. The imaging band path for green channel of JC-M was 520-550 nm ( $\left.\lambda_{\text {ex }}, 490 \mathrm{~nm}\right)$, while that for red channel of JC-A was 570-640 nm $\left(\lambda_{\text {ex }}, 525 \mathrm{~nm}\right)$.

In vivo fluorescent imaging. In vivo fluorescent imaging of TPE-PyT-CPS in HeLa tumor-bearing nude mice model was tested by using the IVIS Lumina III in vivo Imaging System (PerkinElmer). Nude mice at 4-5 weeks old were purchased at pushing medical. TPE-PyT-CPS $(100 \mu \mathrm{M}, 120 \mu \mathrm{L})$ was injected intratumorally and fluorescent images were captured by the Lumina III at $0,3,6,12,18$ and 30 hours after injections (Ex: $530 \mathrm{~nm}, \mathrm{Em}: 690 \mathrm{~nm}$ ). The mice were sacrificed at designated time points $(24 \mathrm{~h})$ and the tissues including heart, liver, spleen, lung, kidney, were excised and imaged by IVIS ${ }^{\circledR}$ Lumina III fluorescence imaging system.

In vivo PDT. HeLa tumors bearing Balb/c mice were randomly divided into four groups $(n=5)$ : saline group (saline light -), saline group with laser irradiation group (saline light +), AlEgen group (AlEgen light -) and AIEgen with laser irradiation group (AIEgen light +). After intra-tumoral injection of TPE-PyT-CPS (0.2 $\mathrm{mM}, 100 \mu \mathrm{L}$ ) for $18 \mathrm{~h}$, the tumor area of the mice was irradiated with $532 \mathrm{~nm}$ laser of $35 \mathrm{~mW} \mathrm{~cm}{ }^{-2}$ for 5 min. The tumor size was measured every 3 days and calculated as follows: volume $=1 / 2$ (tumor length) $\times$ (tumor width) ${ }^{2}$.

Histological analysis. All mice of different groups were sacrificed on day 22, and major organs and tumors were separated and made into slices for H\&E or Tunel staining. ${ }^{[3]}$ Major organs were collected and fixed in $4 \%$ paraformaldehyde, which were then embedded into paraffin, sliced at thickness of $5 \mu \mathrm{m}$. The tissue slices were stained with H\&E or TUNEL and then imaged by optical microscopy and assessed by 3 independent pathologists.

\section{Declarations}

\section{Competing interests}

The authors declare no competing financial interest.

\section{Acknowledgements}


The work was under financial supports from the National Natural Science Foundation of China (21907050, 21731004, 21977044, 91953201), the Natural Science Foundation of Jiangsu Province (BK20202004, BK20190282) and the Excellent Research Program of Nanjing University (ZYJH004).

\section{Author contributions}

M.L., Y.C., W.H. and Z.G. designed the study. M.L. synthesized probes; C. W. performed the DFT calculations. M.L., S. Y. and S. J. performed the spectroscopic and cellular experiments; M.L., Y. G. and H. Y. performed the in vivo experiments; M.L. and Y.C. co-wrote the manuscript, Y.C. and H. F. revised the manuscript. All authors discussed the results and commented on the paper. All authors have given approval to the final version of the manuscript.

\section{References}

(1) Lucky, S. S.; Soo, K. C.; Zhang, Y., Chem. Rev. 2015, 115 (4), 1990-2042.

(2) Noh, I.; Kim, H. O.; Choi, J.; Choi, Y.; Lee, D. K.; Huh, Y. M.; Haam, S., Biomaterials 2015, 53, 763-774.

(3) Luo, S.; Zhang, E.; Su, Y.; Cheng, T.; Shi, C., Biomaterials 2011, 32 (29), 7127-7138.

(4) Li, X.; Kim, C. Y.; Lee, S.; Lee, D.; Chung, H. M.; Kim, G.; Heo, S. H.; Kim, C.; Hong, K. S.; Yoon, J., J. Am. Chem. Soc. 2017, 139 (31), 10880-10886.

(5) Wang, D.; Su, H.; Kwok, R. T. K.; Shan, G.; Leung, A. C. S.; Lee, M. M. S.; Sung, H. H. Y.; Williams, I. D.; Lam, J. W. Y.; Tang, B. Z., Adv. Funct. Mater. 2017, 27 (46), 1704039-1704048.

(6) Hu, F.; Xu, S.; Liu, B., Adv. Mater. 2018, 30 (45), 1801350-180378.

(7) Wu, W.; Mao, D.; Hu, F.; Xu, S.; Chen, C.; Zhang, C. J.; Cheng, X.; Yuan, Y.; Ding, D.; Kong, D.; Liu, B., Adv. Mater. 2017, 29 (33), 1700548-1700554.

(8) Yang, L.; Wang, X.; Zhang, G.; Chen, X.; Zhang, G.; Jiang, J., Nanoscale 2016, 8 (40), 17422-17426.

(9) Nguyen, V. N.; Yan, Y.; Zhao, J.; Yoon, J., Acc. Chem. Res. 2021, 54 (1), 207-220.

(10) Kwok, R. T.; Leung, C. W.; Lam, J. W.; Tang, B. Z., Chem. Soc. Rev. 2015, 44 (13), 4228-4238.

(11) Ethirajan, M.; Chen, Y.; Joshi, P.; Pandey, R. K., Chem. Soc. Rev. 2011, 40 (1), 340-362.

(12) Kamkaew, A.; Lim, S. H.; Lee, H. B.; Kiew, L. V.; Chung, L. Y.; Burgess, K., Chem. Soc. Rev. 2013, 42 (1), 77-88.

(13) Lv, W.; Zhang, Z.; Zhang, K. Y.; Yang, H.; Liu, S.; Xu, A.; Guo, S.; Zhao, Q.; Huang, W., Angew. Chem. Int. Ed. 2016, 55 (34), 9947-9951. 
(14) Chakrabortty, S.; Agrawalla, B. K.; Stumper, A.; Vegi, N. M.; Fischer, S.; Reichardt, C.; Kogler, M.; Dietzek, B.; Feuring-Buske, M.; Buske, C.; Rau, S.; Weil, T., J. Am. Chem. Soc. 2017, 139 (6), 2512-2519.

(15) Luo, S.; Tan, X.; Fang, S.; Wang, Y.; Liu, T.; Wang, X.; Yuan, Y.; Sun, H.; Qi, Q.; Shi, C., Adv. Funct. Mater. 2016, 26 (17), 2826-2835.

(16) Garg, A. D.; Agostinis, P., Photochem. Photobiol. Sci. 2014, 13 (3), 474-487.

(17) Li, W.; Yang, J.; Luo, L.; Jiang, M.; Qin, B.; Yin, H.; Zhu, C.; Yuan, X.; Zhang, J.; Luo, Z.; Du, Y.; Li, Q.; Lou, Y.; Qiu, Y.; You, J., Nat. Commun. 2019, 10 (1), 3349-3364.

(18) Wang, L.; Guan, R.; Xie, L.; Liao, X.; Xiong, K.; Rees, T. W.; Chen, Y.; Ji, L.; Chao, H., Angew. Chem. Int. Ed. 2021, 60 (9), 4657-4665.

(19) Huang, H.; Yu, B.; Zhang, P.; Huang, J.; Chen, Y.; Gasser, G.; Ji, L.; Chao, H., Angew. Chem. Int. Ed. 2015, 54 (47), 14049-14052.

(20) Zhou, Z.; Liu, J.; Huang, J.; Rees, T. W.; Wang, Y.; Wang, H.; Li, X.; Chao, H.; Stang, P. J., Proc. Natl. Acad. Sci. US A. 2019, 116 (41), 20296-20302.

(21) Bu, Y.; Xu, T.; Zhu, X.; Zhang, J.; Wang, L.; Yu, Z.; Yu, J.; Wang, A.; Tian, Y.; Zhou, H.; Xie, Y., Chem. Sci. 2020, 11 (37), 10279-10286.

(22) Zhang, W.; Huang, Y.; Chen, Y.; Zhao, E.; Hong, Y.; Chen, S.; Lam, J. W. Y.; Chen, Y.; Hou, J.; Tang, B. Z., ACS. Appl. Mater. \& Inter. 2019, 11 (11), 10567-10577.

(23) Zappa, F.; Failli, M.; De Matteis, M. A., Curr. Opin. Cell. Biol. 2018, 50, 102-116.

(24) Hicks, S. W.; Biochim. Biophys. Acta. 2005, 1744 (3), 406-414.

(25) Maag, R. S.; Hicks, S. W.; Machamer, C. E., Curr. Opin. Cell. Biol. 2003, 15 (4), 456-461.

(26) Nakagomi, S.; Barsoum, M. J.; Bossy-Wetzel, E.; Sütterlin, C.; Malhotra, V.; Lipton, S. A., Neurobiol. Dis. 2008, 29 (2), 221-231.

(27) Fan, J.; Hu, Z.; Zeng, L.; Lu, W.; Tang, X.; Zhang, J.; Li, T., Int. J. Dev. Neurosci. 2008, 26 (6), 523-534.

(28) Hu, Z.; Zeng, L.; Huang, Z.; Zhang, J.; Li, T., Neurochem. Res. 2007, 32 (8), 1265-1277.

(29) Xu, S.; Yuan, Y.; Cai, X.; Zhang, C. J.; Hu, F.; Liang, J.; Zhang, G.; Zhang, D.; Liu, B., Chem. Sc.i 2015, 6 (10), 5824-5830.

(30) Wu, W.; Mao, D.; Xu, S.; Ji, S.; Hu, F.; Ding, D.; Kong, D.; Liu, B., Mater. Horizons. 2017, 4 (6), 1110-1114.

(31) Chisholm, D. R.; Hughes, J. G.; Blacker, T. S.; Humann, R.; Adams, C.; Callaghan, D.; Pujol, A.; Lembicz, N. K.; Bain, A. J.; Girkin, J. M.; Ambler, C. A.; Whiting, A., Org. Biomol. Chem. 2020, 18 (45), 9231-9245. 
(32) Ramirez, I. B.; Lowe, M., Semin. Cell. Dev. Biol. 2009, 20 (7), 770-779.

(33) Gillingham, A. K.; Munro, S., Trends. Cell. Biol. 2016, 26 (6), 399-408.

(34) Ghaemi, B.; Moshiri, A.; Herrmann, I. K.; Hajipour, M. J.; Wick, P.; Amani, A.; Kharrazi, S., ACS. Appl. Mater \& Inter. 2019, 11 (50), 46408-46418.

(35) Lesa, G. M.; Seemann, J.; Shorter, J.; Vandekerckhove, J.; Warren, G., J. Biol. Chem. 2000, 275 (4), 2831-2836.

(36) How, P. C.; Shields, D., J. Biol. Chem. 2011, 286 (10), 8565-8576.

(37) Chiu, R.; Novikov, L.; Mukherjee, S.; Shields, D., J. Cell. Biol. 2002, 159 (4), 637-648.

(38) Ming, L.; Wang, P.; Bank, A.; Yu, J.; Zhang, L., J. Biol. Chem. 2006, 281 (23), 16034-16042.

(39) Lopez, J.; Tait, S. W., Br. J Cancer. 2015, 112 (6), 957-962.

(40) Redza-Dutordoir, M.; Averill-Bates, D. A., Biochim. Biophys. Acta. 2016, 1863 (12), $2977-2992$.

(41) Zhang, S.; Huang, Q.; Zhang, L.; Zhang, H.; Han, Y.; Sun, Q.; Cheng, Z.; Qin, H.; Dou, S.; Li, Z., Nanoscale 2018, 10 (7), 3130-3143.

\section{Figures}

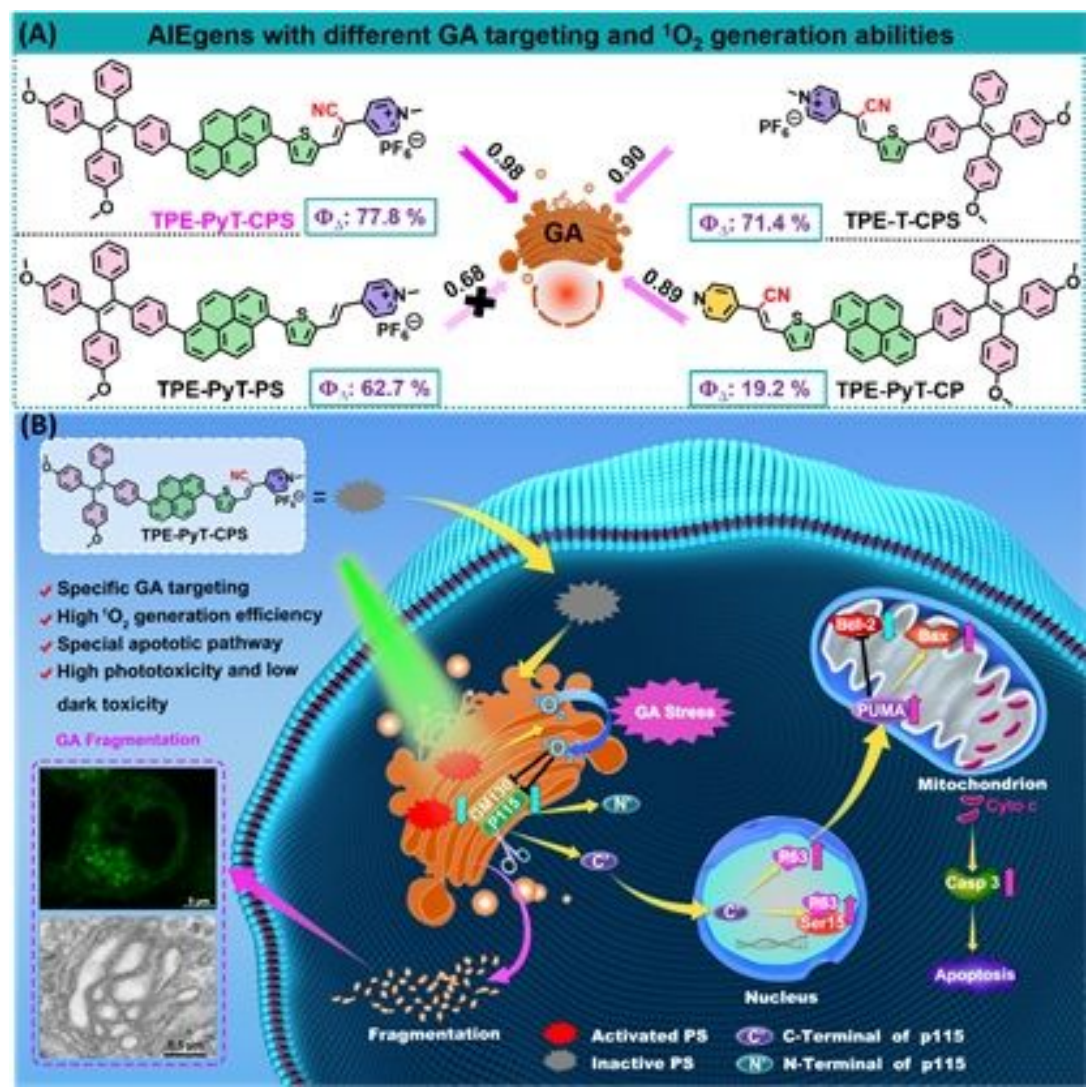


(A) The chemical structures of AlEgens with different GA targeting (PCC values) and ROS generation abilities. (B) Schematic illustration of the GA targeting AlEgen induced GA stress and the crosstalk of GA and mitochondria for cell apoptosis upon PDT.
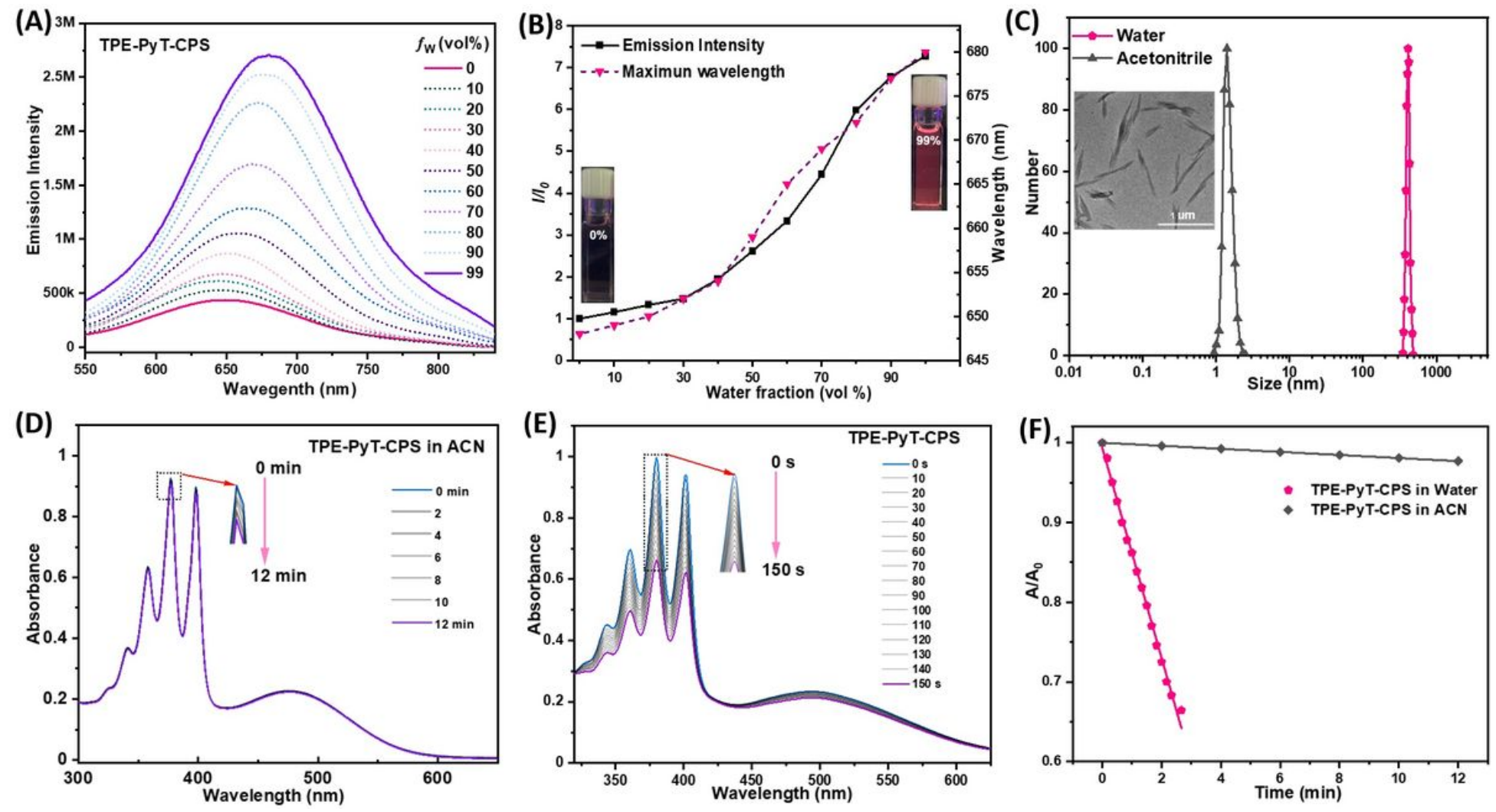

\section{Figure 2}

AIE property of TPE-PyT-CPS and its ROS generation ability in different solvents. (A) Emission spectra of TPE-PyT-CPS in acetonitrile/water mixtures with varied water fractions ( $\mathrm{fw}$ ). (B) Plot of $\mathrm{I} / \mathrm{IO}$ (dark dot) and the maximum emission wavelength (red dot) of TPE-PyT-CPS in different fw of the solvent mixture, 10 represents the emission intensity in acetonitrile. (C) Particle sizes of TPE-PyT-CPS $(10 \mu \mathrm{M})$ in water and acetonitrile dynamic light scattering, Inset: TEM images of TPE-PyT-CPS in water (D), (E) Changes of UVvis spectra of ABDA $(50 \mu \mathrm{M})$ in the presence of TPE-PyT-CPS $(10 \mu \mathrm{M})$ in acetonitrile and water under different durations of light irradiation ( $25 \mathrm{~mW} \mathrm{~cm}-2)$. (F) The decomposition rates of ABDA in different solvent with TPE-PyT-CPS under light irradiation, respectively.
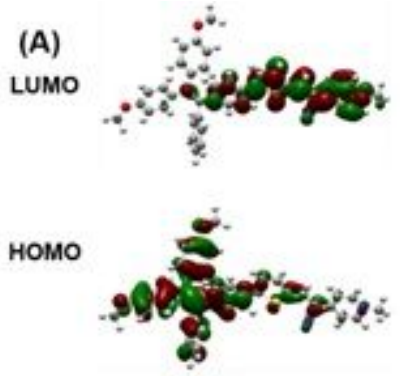

TPE-T-CPS
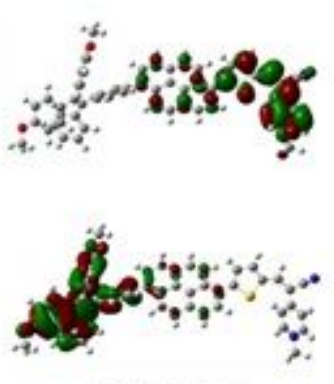

TPE-PYT-CPS

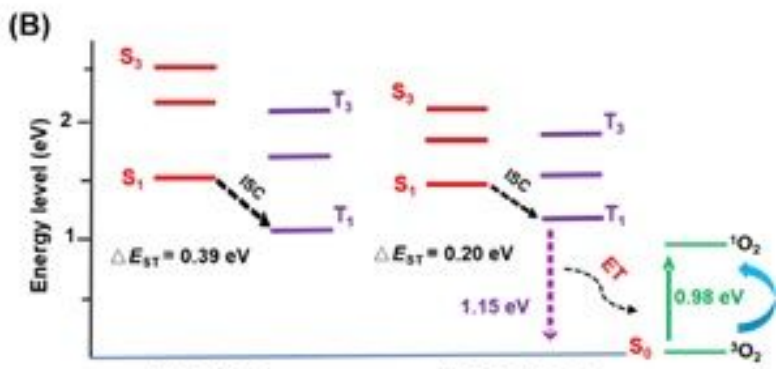

TPE-T-CPS

TPE-PYT-CPS 


\section{Figure 3}

Calculated frontier molecular orbitals and energy levels for TPE-T-CPS and TPE-PyT-CPS. (A) HOMOLUMO electronic cloud distributions of TPE-T-CPS and TPE-PyT-CPS by DFT calculations. (B) Energy levels (S1-S3, T1-T3) of TPE-T-CPS and TPE-PyT-CPS calculated by the vertical excitation of the optimized structures.

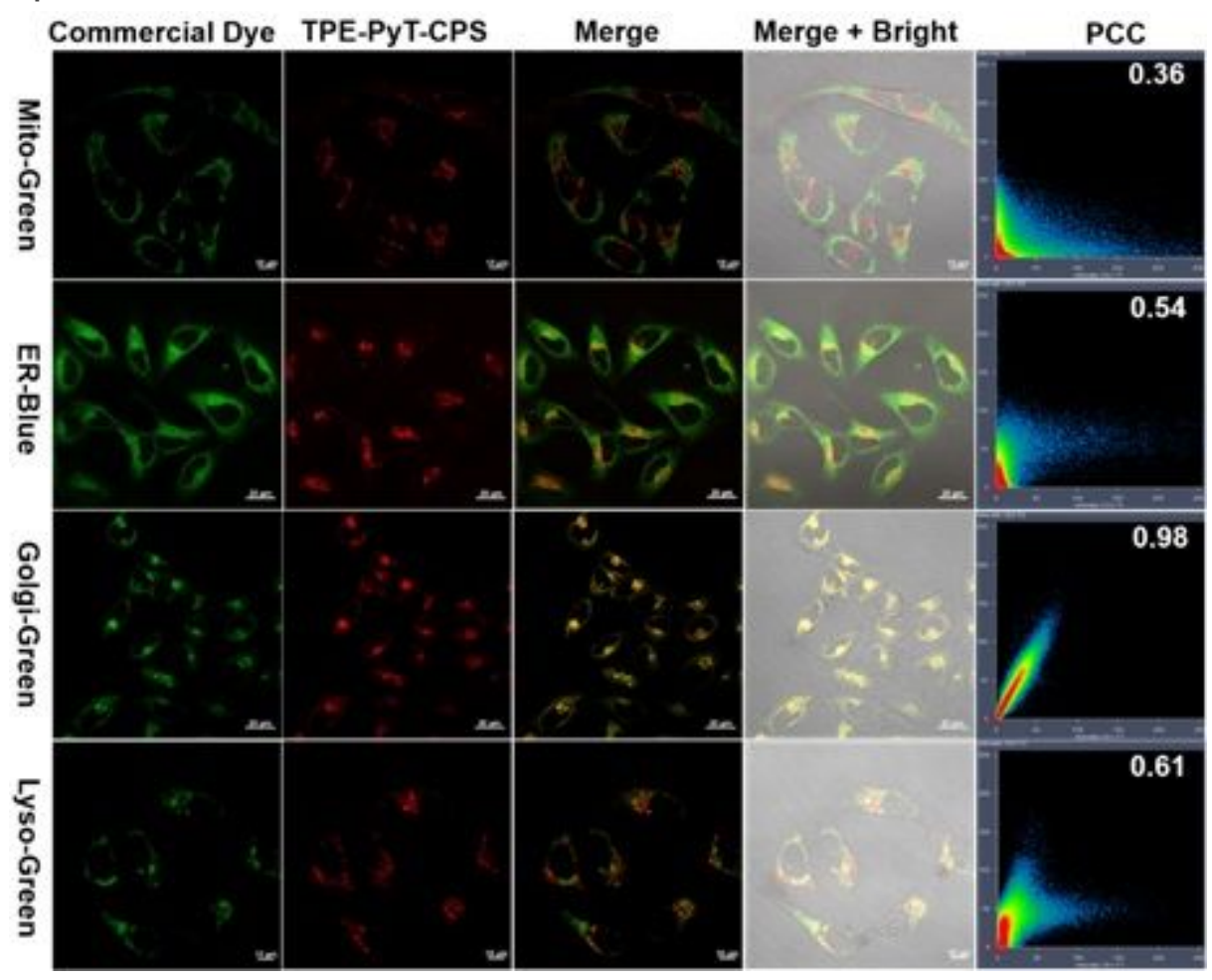

Figure 4

Subcellular co-localization of TPE-PyT-CPS. CLSM of HeLa cells stained with different commercial probes

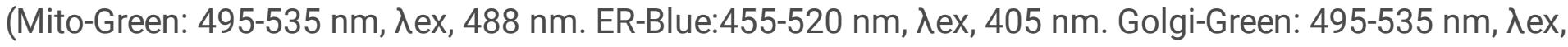
$488 \mathrm{~nm}$. Lyso-Green:495-535 nm, $\lambda$ ex, $488 \mathrm{~nm})$ and TPE-PyT-CPS (10 $\mu \mathrm{M}, 600-750 \mathrm{~nm}, \lambda e x, 488 \mathrm{~nm})$. Scale bar: $20 \mu \mathrm{m}$ for ER and Golgi channel, $10 \mu \mathrm{m}$ for Mito and Lyso channel. 
(A)
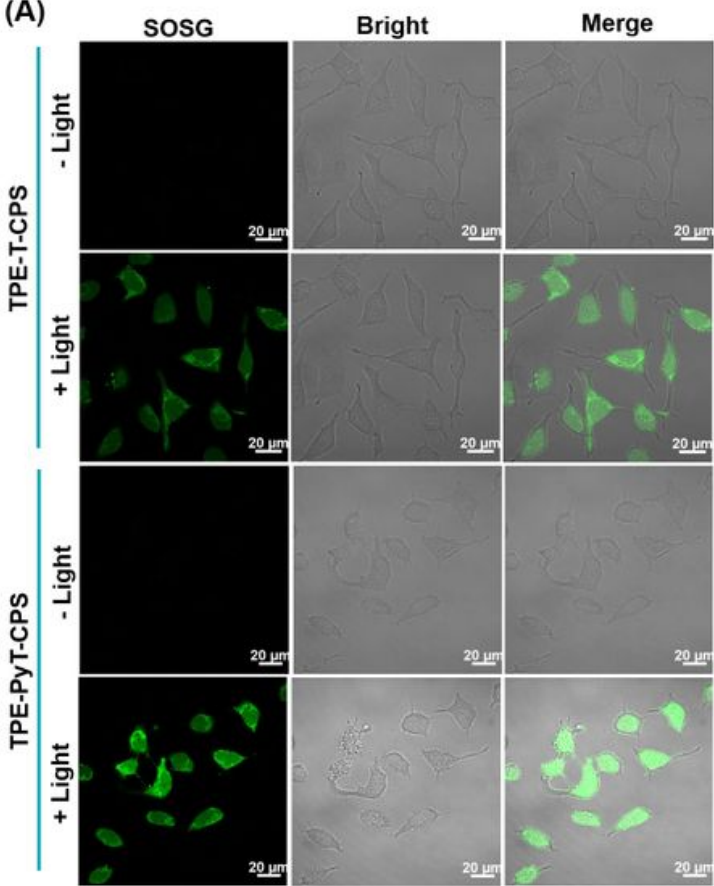

(B)

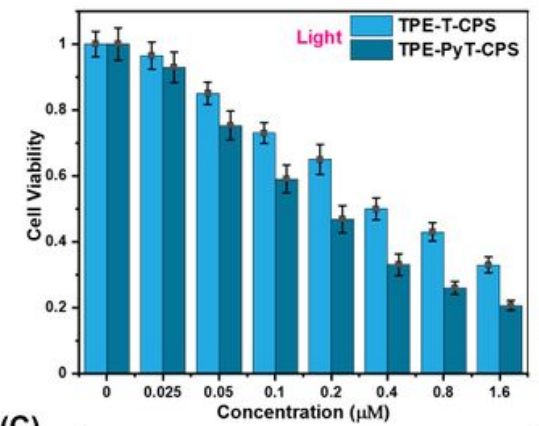

(C)

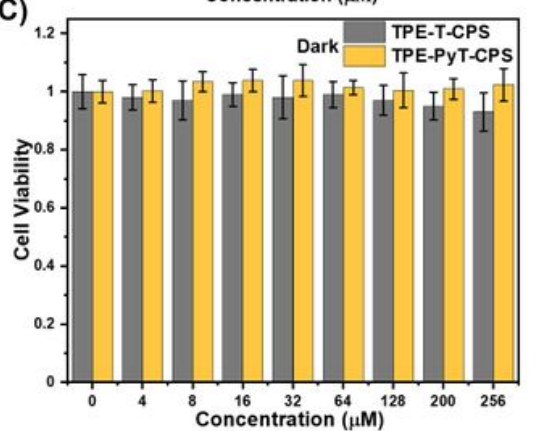

(D) + Ligh
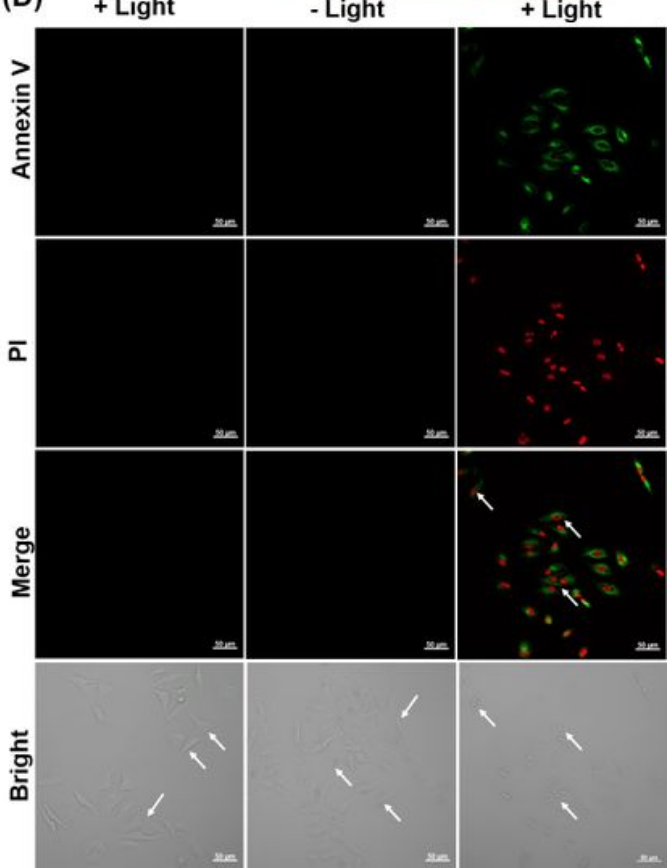

\section{Figure 5}

Intracellular ROS, cytotoxicity and cells apoptosis induced by PDT. (A) Intracellular 102 detection by CLSM after HeLa cells were incubated with TPE-PyT-CPS (0.2 $\mu \mathrm{M})$ and SOSG under laser irradiations (25 $\mathrm{mW}$ cm-2 for 2 min, scale bar: $20 \mu \mathrm{m})$. Cell viabilities of HeLa cells after incubation with varied concentrations of TPE-PyT-CPS (B) with or (C) without $532 \mathrm{~nm}$ laser irradiation (65 mW cm-2, $2 \mathrm{~min})$. (D) Apoptosis of HeLa cells treated with TPE-PyT-CPS $(0.2 \mu \mathrm{M})$ under $532 \mathrm{~nm}$ laser $(65 \mathrm{~mW} \mathrm{~cm}-2$ for $2 \mathrm{~min}$, scale bar: $50 \mu \mathrm{m})$.

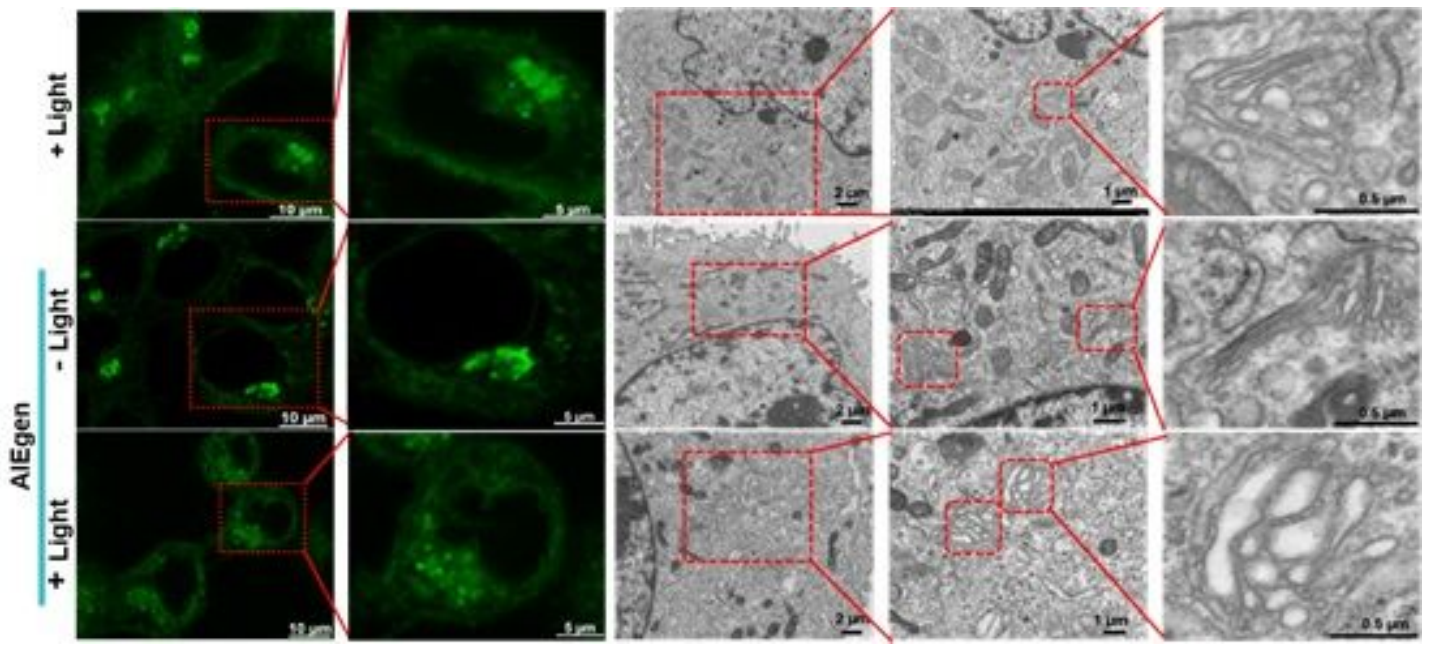

Figure 6

Morphological changes of Golgi apparatus observed by CLSM (stained with Gogli Green) and TEM imaging in HeLa cells after treatment with light only, TPE-PyT-CPS (0.2 $\mu \mathrm{M})$ with or without light irradiation (25 mW cm-2, $2 \mathrm{~min}$ ). 

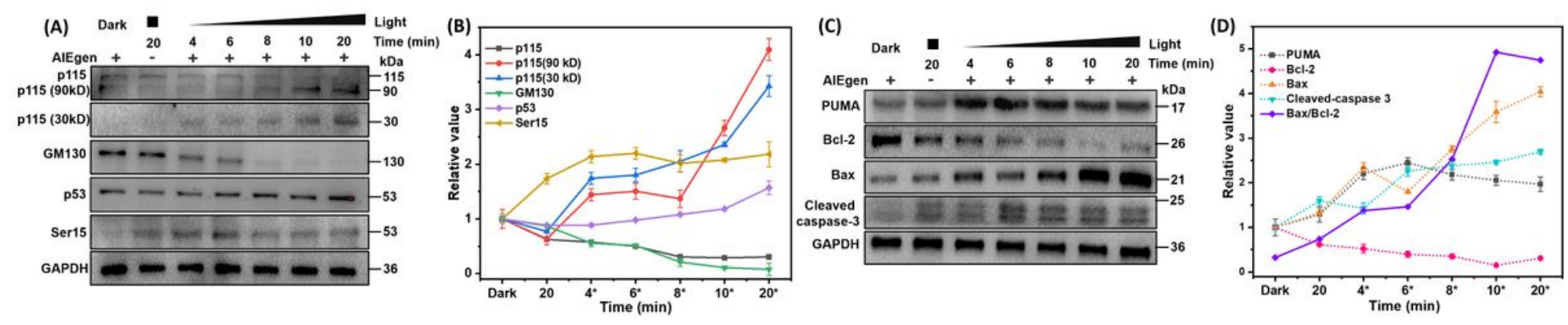

\section{Figure 7}

Effect of PDT on Golgi apparatus and mitochondria associated protein expression. The expressions of p115, p115 (90 kD), p115 (30kD), GM130, p53, ser15 in Golgi apparatus (A) and PUMA, Bcl-2, Bax, caspase-3 in mitochondria (C) after different treatment with TPE-PyT-CPS $(0.2 \mu \mathrm{M})$ under light irradiation (25 mW cm-2, $2 \mathrm{~min}$ ). (B) and (D) normalized protein expression changes for different irradiation time, the protein expression in the dark was taken as 1.

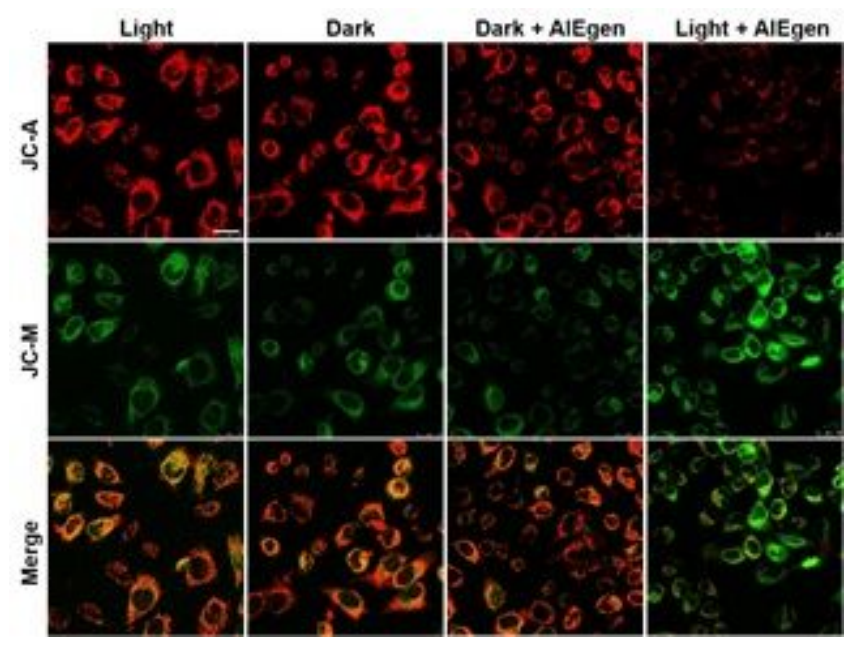

\section{Figure 8}

Confocal fluorescence imaging of MMP in HeLa cells in the absence and presence of TPE-PyT-CPS (0.2 $\mu \mathrm{M})$ via JC-1 staining assay under different conditions. Scale bar: $25 \mu \mathrm{m}$. 


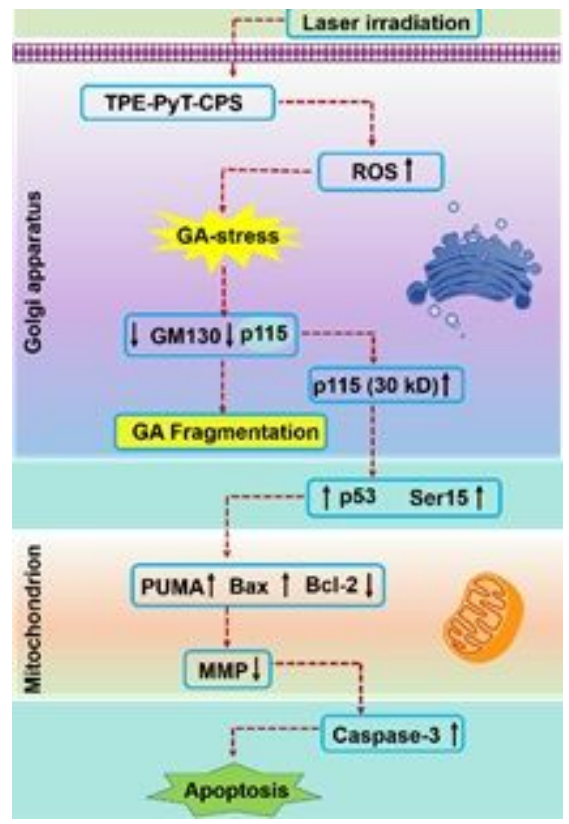

\section{Figure 9}

Proposed mechanism for the cell apoptosis through the crosstalk between Golgi apparatus and mitochondria in GA-targeted photodynamic therapy.

(A)
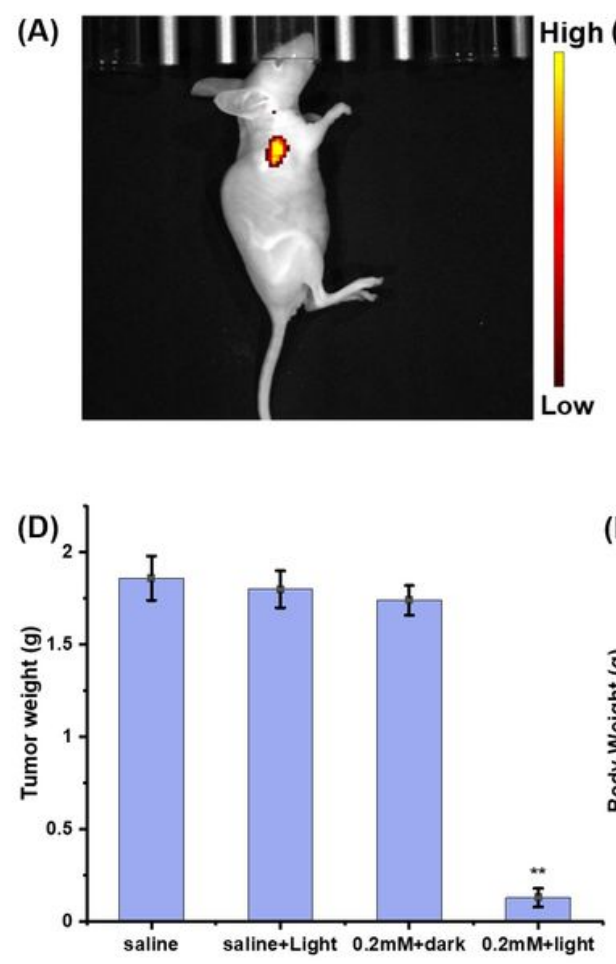

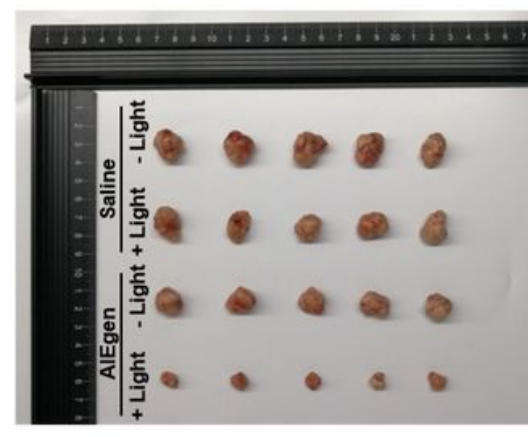

(C)

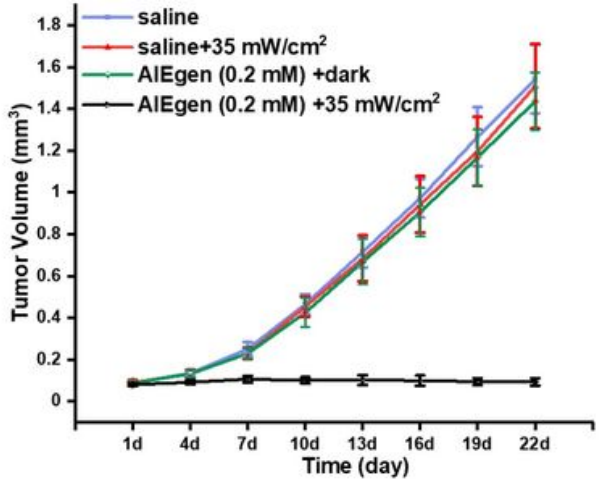

(F)
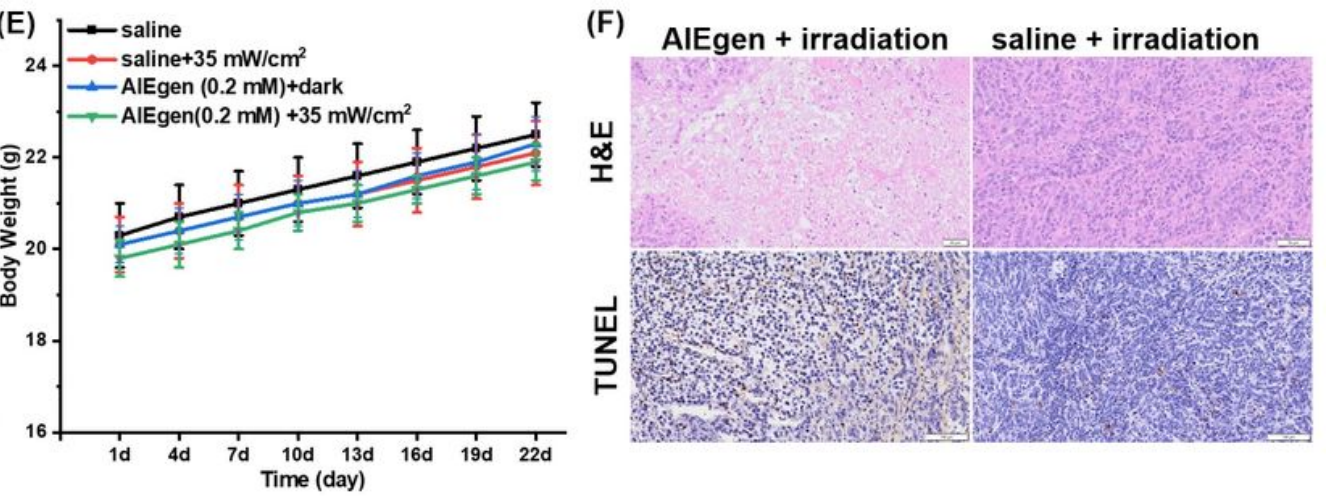

Figure 10

Therapeutic effect of TPE-PyT-CPS in vivo. (A) Fluorescent image of a mouse after intratumoral injection of TPE-PyT-CPS $(0.1 \mathrm{mM}, 120 \mu \mathrm{L})$ for $18 \mathrm{~h}$. (B) Photographs of excised tumors on the 22th day. (C) Changes of tumor volume and weight (D) after PDT. (E) Body weight curves of mice during PDT 
treatment employing TPE-PyT-CPS $(0.2 \mathrm{mM}, 120 \mu \mathrm{L})$ as the PS and 532 laser irradiations of $35 \mathrm{~mW} \mathrm{~cm}-2$ for 5 min at different time points post-treatment in different groups: saline (light -), saline (light +), TPEPyT-CPS (light -) and TPE-PyT-CPS (light +) groups. (F) H\&E staining (scale bar: $50 \mu \mathrm{m}$ ) and apoptotic analysis of TUNEL staining (scale bar: $100 \mu \mathrm{m}$ ) images of tumor slices from mice after treatment with saline and TPE-PyT-CPS under 532 laser irradiations.

\section{Supplementary Files}

This is a list of supplementary files associated with this preprint. Click to download.

- SupportingInformationofNC.docx 